\title{
QUEUEING MODELS IN MOBILE AD-HOC NETWORKS
}

\author{
Baskar, S. and S. Palaniammal \\ Department of Science and Humanities, \\ Sri Krishna College of Technology, Kovaipudur, Coimbatore-641 042, Tamil Nadu, India
}

Received 2013-09-25; Revised 2013-11-24; Accepted 2013-12-26

\begin{abstract}
The Mobile Ad-hoc Networks (MANETs) has gain an essential part of the attention of researchers and become very well-liked in last few years. MANETs can operate with no fixed communications and can live rapid changes in the network topology. They can be studied officially as graphs in which the set of boundaries varies in time. One of the main methods to determine the presentation of MANETs is simulation. This study proposes Enhanced Probabilistic Adhoc on Demand Distance Vector (EPAODV) routing protocol, which solves the broadcast storm problem of Adhoc on Demand Distance Vector (AODV). Our evaluation of MANETs is based on the evaluation of the throughput, end to end delay and packet delivery ratio. We evaluated the end to end delay as it is one of the most important characteristic evaluation metric in computer networks. In our proposed algorithm, using a queueing model M/M/C: $\infty / \mathrm{FIFO,} \mathrm{we} \mathrm{are} \mathrm{able} \mathrm{to} \mathrm{enhance} \mathrm{that} \mathrm{better} \mathrm{results} \mathrm{are}$ obtained in the case EPAODV protocol such as increasing throughput, data delivery ratio and then decreasing the end delay compare to the existing protocols.
\end{abstract}

Keywords: Mobile Adhoc Networks (MANETs), Enhanced Probabilistic Adhoc On Demand Distance Vector (EPAODV), Adhoc On-Demand Distance Vector (AODV) Routing Protocol, Probabilistic Adhoc On Demand Distance Vector (PAODV), Queueing Model

\section{INTRODUCTION}

A mobile ad-hoc network is formed by mobile hosts. As nodes in MANETs are mobile, so the link failure and re-establishment of routes takes place frequently. The mobility of nodes imply that the routing protocols of MANETs have to cope with frequent topology changes while attempting to create correct routing tables. A topology of 18 nodes can be viewed in Fig. 1. Because the nodes are mobile, the topology keeps changing which makes the transfer of packets complicated. To achieve this two types of routing protocols have been proposed: proactive routing protocol (table-driven) and reactive (or on-demand) routing protocol or hybrid of the both. Proactive routing protocols give fast reply to topology changes by incessantly monitoring topology changes and disseminating the related information as needed over the network. Reactive (On-Demand) routing protocols finds a route on demand by flooding the network with route request packets. The main disadvantage of such algorithms are the respective amount of data for maintenance and slow reaction on restructuring and failures. Example of pro-active algorithm is Wireless Routing Protocol (WRP) which used an enhanced version of the distance-vector routing protocol that uses the classical Bellman-Ford algorithm to calculate paths. Reactive (on-demand) routing protocols find a route on demand by flood the network with route request packets. The main disadvantages of such algorithms are elevated latency time in direction finding and extreme flooding can lead to network blockage.

AODV does not preserve the topological in order about the whole network and constructs a route when it is required. When a source node needs a route to some destination it broadcasts a Route Request (RREQ) packets to its immediate neighbours. Each neighboring node rebroadcasts the received RREQ packet only once if it has no valid route to the destination. Table 1 shows the parameters are using in our work, we have take an different protocols, Transmission range and Packet size based data transmission on the network.

Corresponding Author: Baskar, S., Department of Science and Humanities, Sri Krishna College of Technology, Kovaipudur, Coimbatore-641 042, Tamil Nadu, India 


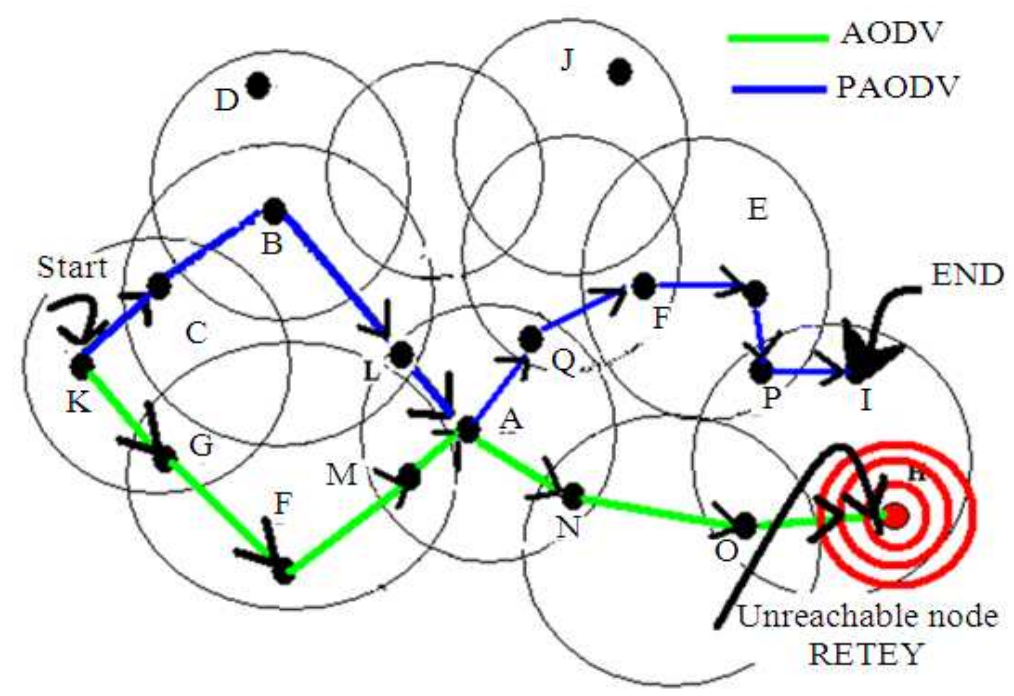

Fig. 1. Routing between AODV and PAODV

Table 1. Simulation parameters

\begin{tabular}{ll}
\hline Parameter & Value \\
\hline Protocols studied & Enhanced PAODV with \\
& Probability (EPAODV) \\
Number of node & 71 \\
Simulation time & $20 \mathrm{sec}$ \\
Simulation area & $1000 \times 1000 \mathrm{~m}$ \\
Transmission range & $250 \mathrm{~m}$ \\
Node movement model & Random Way Point \\
Traffic model & CBR (UDP) \\
Transfer per packet & 512 Bytes \\
\hline
\end{tabular}

Each intermediate node that participates in forwarding the Route Reply (RREP) packet creates a forward route pointing towards the destination. Such protocol needs high over head in the attendance of high mobility and great number of nodes. Also it considerably limits its viability. Therefore the available deterministic protocols can carry out the routing process only under a group of restricted constraints on the network coverage area size, node density or mobility. Probabilistic protocols, on the other hand, about forecast the network topology using only probabilistic and traceable information. Therefore they can considerably reduce the amount of overhead in contrast with the deterministic protocols and thus are more scalable and topology self-governing.

This study reports the following main contributions. First it proposes a routing protocol called Enhanced probabilistic adhoc on-demand routing protocol which reduces the end delay compared with existing probabilistic routing protocol. Second the MANETs characteristics throughput, data delivery ratio, end to end delay has been calculated. Finally the results have been compared with the existing probabilistic routing protocol results. Here our model EPAODV enhances the exiting Probability based Distance Vector direction-finding algorithm (PAODV) by changing queueing model which is more effective by using the new EPAODV routing algorithm which determines the performance of the networks. The probability of a node being stable in the path is modeled by queueing theory where the stability of a node is measured by number of packets arrived at a node and the number of packets being serviced by the node per unit time.

Khandelwal et al. (2011) proposed a probablistic routing protocol (PAODV) by introducing a queueing model for infinite packet arrivals at a node and they have established that in all aspects such as node density, coverage area size and node mobility of networks characteristics of PAODV is more efficient than AODV. The proposed routing protocol uses $\mathrm{M} / \mathrm{M} / \mathrm{C}: \infty / \mathrm{FIFO}$ queueing model for each node in the data transmission path so that it can transmit the packets to the destination quickly. The results have been taken for throughput, data delivery ratio and then end delay. Comparing results of EPAODV with existing PAODV, the consequences demonstrate that our EPAODV protocol increases throughput, data delivery ratio and then decreases the end delay.

The remainder of the study is organized as follows:

- Section 1: Introduction 
- Section 2: The related research in MANETs routing algorithms

- Section 3: Assumptions and the proposed algorithms

- Section 4: Performance analysis

- Section 5: The simulation run

- Section 6: Analysis and results of simulation runs

- Section 7: Conclusion

\section{RELATED WORK}

From the literature survey it is well known that in the Mobile Ad-hoc Network (MANETs) the competence of broadcasting protocol can radically affect the performance of the whole network. Appropriate use of a probabilistic method can reduce the number of rebroadcasting and hence reduce the chance of contention and collision among neighboring nodes. A high-quality probabilistic broadcast protocol can achieve higher throughput and lower energy consumption, without sacrifice the reachability or having any important poverty.

The significant and outstanding work by (Yassein and Khaoua, 2007) is worth mentioning here since it contributes a lot to the development of research in MANETs routing protocol.

Yassein and Khaoua (2007) the applications of probabilistic flooding in MANETs by evaluating the performance of familiar Probabilistic flooding by compare it to "blind" flooding as used with the Ad Hoc on Demand Distance Vector (AODV) routing protocol as well as a fixed probabilistic approach. The results show that the Adjusted Probabilistic flooding exhibits superior performance in terms of both reachability and save rebroadcasts.

Yassein et al. (2010) proposed a Smart Probabilistic Broadcasting (SPB) scheme as a new probabilistic method to improve the presentation of exiting ondemand routing protocols by dropping the RREQ overhead during the rout discovery process. The simulation result shows that the combination of AODV and a appropriate probabilistic route discovery can reduce the average end-to-end delay as well as overhead, while achieving low normalize directionfinding load, compared to AODV that uses fixed probability and blind flooding.

\section{PROPOSED ALGORITHM}

The literature survey shows that the probabilistic route discovery plays a prominent role in Manets routing protocol. The proposed algorithm gives importance to queueing models. We relate our proposed algorithm to $\mathrm{M} / \mathrm{M} / \mathrm{C}$ : ( $\infty / \mathrm{FIFO})$ queueing model. The arriving pockets can be regarded as the arriving customers in an unbounded queue and the route finding algorithm as the service provided to these customers (packets). According to the FIFO model, the traffic arrives randomly, which can be treated as Poisson distribution and are served in FIFO basis, which is an exponential distribution. In our queueing model the first $M$ represents Markovian exponential distribution of inter-arrival times, second $M$ represents Markovian exponential distribution of service times, $C$ (a positive integer) represents the number of servers in a queueing system. In our model there are unlimited number of customers enter.

Here we consider $\mathrm{C}$ number of servers. The arrival rate is $\lambda$ and the service rate per service channel is $\mu$. The effect of using $C$ parallel service channels is a proportionate increase in the service rate of the facility to $n \mu$ if $n \leq C$ and $C \mu$ if $n>C$. Thus the steady state probability that there will exist ' $n$ ' or greater than ' $n$ ' number of packets at a given time in a system is given by:

$$
\begin{aligned}
& \mathrm{p}_{\mathrm{n}}=\left\{\begin{array}{c}
\frac{\gamma^{\mathrm{n}} \mathrm{P}_{0}}{\mathrm{n} \mu(\mathrm{n}-1) \mu \ldots .(1) \mu} ; 1 \leq \mathrm{n} \leq \mathrm{C} \\
\frac{\gamma^{\mathrm{n}} \mathrm{P}_{0}}{(\mathrm{C} \mu)(\mathrm{C} \mu) \ldots .(\mathrm{C} \mu)(\mathrm{C} \mu)(\mathrm{C}-1) \mu(\mathrm{C}-2) \mu \ldots(1) \mu} ; \mathrm{n} \geq \mathrm{C}
\end{array}\right. \\
& =\left\{\begin{array}{l}
\frac{\lambda^{n} P_{0}}{n ! \mu^{n}} \text { if } 1 \leq n \leq C \\
\frac{\lambda^{n} P_{0}}{C^{n-c} C ! \mu^{n}} \text { if } 1 \leq n \leq C
\end{array}=\left\{\begin{array}{l}
\frac{1}{n} \rho^{n} P_{0} \text { if } 1 \leq n \leq C \\
\frac{1}{C^{n-c} C !} \rho^{n} P_{0} \text { if } n>C
\end{array}\right.\right.
\end{aligned}
$$

where, $\rho=\frac{\gamma}{C \mu}$ To find the value of $\mathrm{P}_{0}$, we use the boundary condition $\sum_{\mathrm{n}=0}^{\infty} \mathrm{P}_{\mathrm{n}}=1$. Thus:

$$
\begin{aligned}
& \sum_{n=0}^{C-2} P_{n}+\sum_{n=C}^{\infty} P_{n}=1\left[\sum_{n=0}^{C-1} \frac{1}{n} \rho^{n}+\sum_{n=C}^{\infty} \frac{1}{C^{n-c} C !} \rho^{n}\right] P_{0}=1 \\
& P_{0}=\left[\sum_{n=0}^{C-1} \frac{1}{n !} \rho^{n}+\rho^{C} \sum_{n=C}^{\infty}\left(\frac{\rho}{C}\right)^{n-C} \frac{1}{C !}\right]^{-1} \\
& P_{0}=\left[\sum_{n=0}^{C-1} \frac{1}{n} !\left(\frac{\lambda}{\mu}\right)^{n}+\frac{1}{C !}\left(\frac{\lambda}{\mu}\right)^{C} \frac{C \mu}{C \mu-\lambda}\right]^{-1}
\end{aligned}
$$


Thus in order to decide whether a packet at a node should be forwarded to another node on a particular route is decided by considering the strength of that route, which in turn in decided by the probability function described above in Equation (1).

The proposed algorithm is a combination of the probability based schemes and knowledge based schemes and automatically adjust the re-broadcast chance at each mobile node according to the price of the immediate number of neighbors in its transmission range.

To select the value of probability $p$, the average neighbor number is analyzed. Let $\mathrm{X}$ be area of the adhoc network, $\mathrm{n}$ be the total number of mobile nodes in the network and ' $r$ ' be the radio transmission range then average number of nodes ( $\mathrm{n}$ bar) can be computed from the formula Equation (2):

$$
\overline{\mathrm{n}}=\frac{(\mathrm{n}-1) \mathrm{pr}^{2}}{\mathrm{X}}
$$

The value of fixed probability that used in AODVFixed Point is set at $p=0.8$. It has been shown that this probability value enable fixed probabilistic flooding to achieve a good performance. The brief outline of the proposed algorithm is shown in Fig. 3.

\section{PERFORMANCE ANALYSIS}

In the proposed algorithm our objective is to assess the presentation of the enhanced prospect based ahoc on-demand distance vector by mobile adhoc networks explained in Fig. 2. To high light the perform of a flow diagram is also given in the Fig. 3. The simulation location is formed in Network Simulator (NS2), a network imitation to give suggestion to mobile adhoc networks. Network Simulator (NS2) consists of two key languages: $\mathrm{C}++$ and Object-Oriented Tool Command Language (OTCL). While the $\mathrm{C}++$ define the internal mechanism (that is a back end) of the recreation substance, the OTCL sets up simulation by assemble and configuring the objects as well as scheduling separate events(that is a frontend). The $\mathrm{C}++$ and the OTCL are linked jointly using Tool Command Language (TCL). The simulation has been carried out with 71 wireless movable nodes over a model area of $1000 \times 1000 \mathrm{~m}$ for $20 \mathrm{sec}$ of simulation time. The radio and IEEE 802.11 (MAC) Media Access Control layer model were used. Nodes in our imitation move according to chance way point mobility model, which is casual way with maximum speed from $0 \mathrm{~m} \mathrm{sec}^{-1}$ to $20 \mathrm{~m} \mathrm{sec}^{-1}$. A free gap broadcast direct is indefinite for the simulation. The performance of the protocols was measured with respect to metrics like packet throughput, delivery ratio, delay in simulation of wireless network consists of 71 nodes. The layer nodes in our imitation move according to chance waypoint mobility model, it is a simple direction with maximum speed from $0 \mathrm{~m} \mathrm{sec}^{-1}$ to $20 \mathrm{~m} \mathrm{sec}^{-1}$. A free gap broadcast channel is assumed for the simulation. The replication experiments do not explain for the simplicity shaped multicast members. Multicast network source establish and end sending packets; each packet has a steady size of 512 bytes. The mobile node in network start its trip from an opening place to a random end with a casual selected velocity.

Our reproduction is to examine the performance of the PAODV with Enhanced probability (EPAODV). The simulation environment is created in NS2. It is a network simulator that provides support for simulating multihop wireless networks. NS2 consists of OTCL and $\mathrm{C}++$. The $\mathrm{C}++$ substance are map to OTCL handles using TCL. To sprint a reproduction, we should describe a network state in a TCL imitation script and feeds this script as an input to executable file NS. During the simulation, the packet flow in order can be collected during text-based tracing or Nodal Admittance Matrix (NAM) tracing. After the model, an AWK program (AWK is a language for processing text files) can be use to examine a text-based trace file. The Nodal Admittance Matrix (NAM) program, on extra hand, utilizes a Nodal Admittance Matrix (NAM) trace file to repeat the network simulation using animation.

\subsection{The Performance of EPAODV on Network Parameters}

\subsubsection{The Performance of EPAODV for Flow Diagram}

Let the source and destination nodes be $\mathrm{A}$ and $\mathrm{M}$ respectively. Let node $\mathrm{K}$ move out from its current position and the probability factor of node $\mathrm{K}$ be less than the threshold value. Probability of each node is computed using Equation 1. The actual packet transmission path is:

$$
\mathrm{A} \rightarrow \mathrm{H} \rightarrow \mathrm{I} \rightarrow \mathrm{D} \rightarrow \mathrm{J} \rightarrow \mathrm{K} \rightarrow \mathrm{M}
$$




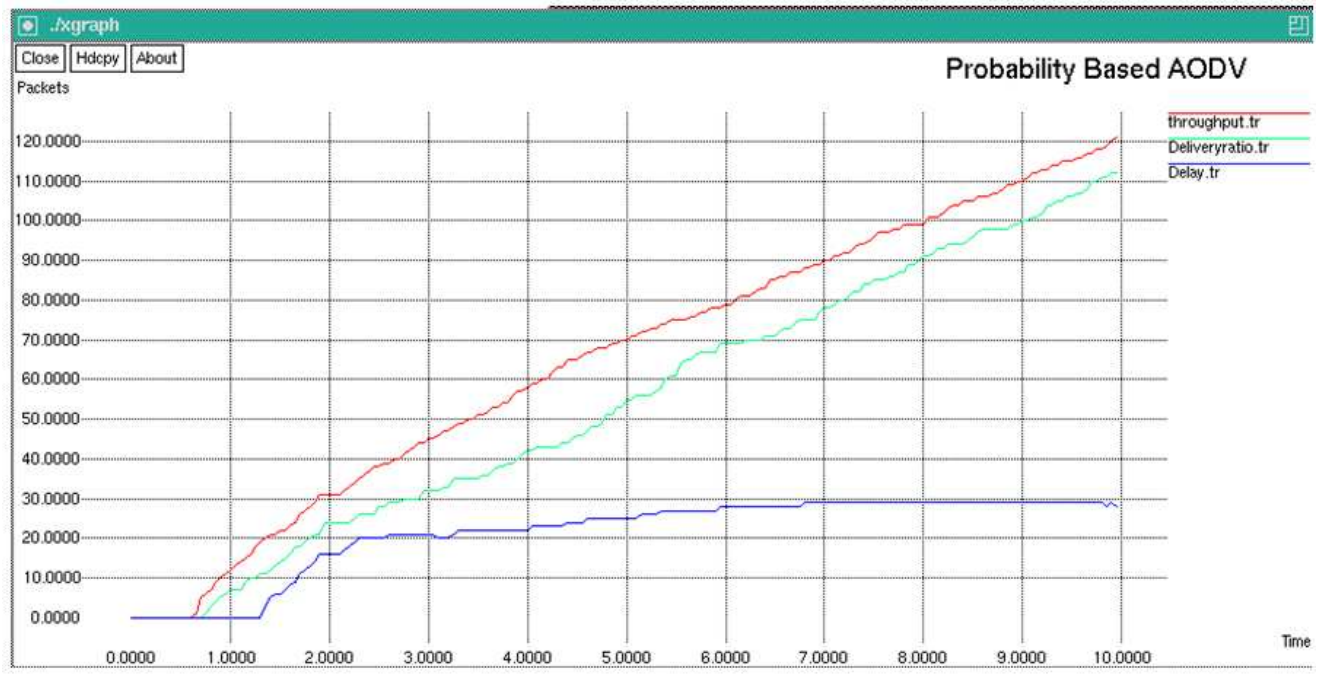

Fig. 2. Performance of probability based AODV

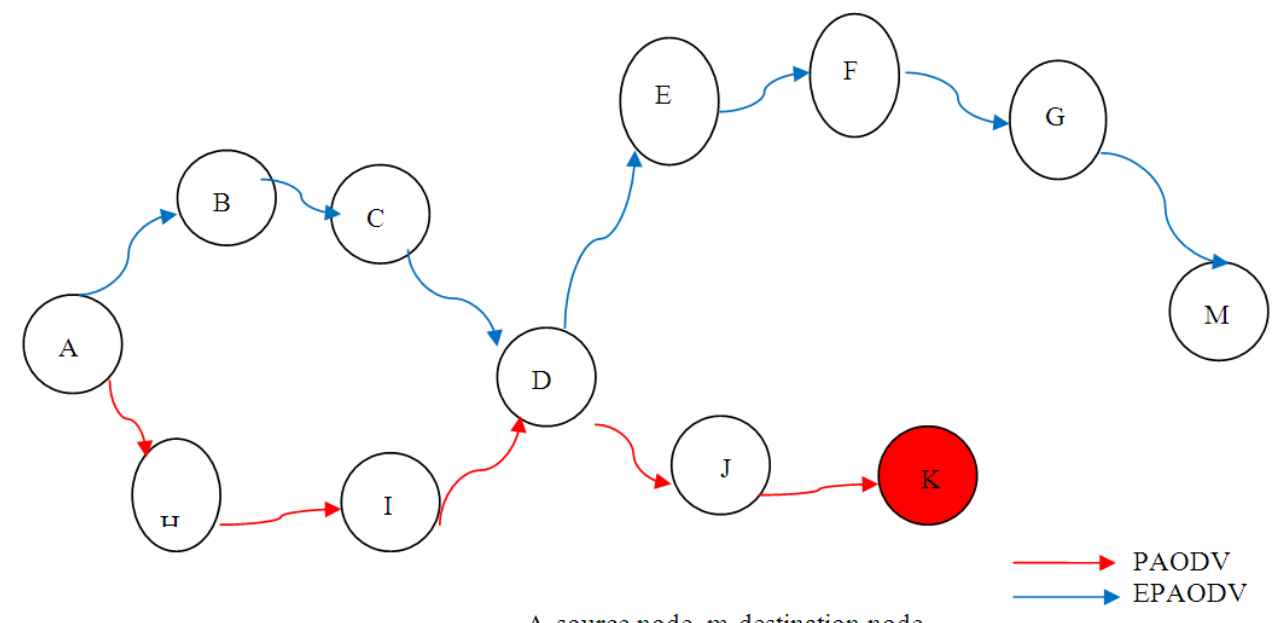

Fig. 3. PAODV and EPAODV routing protocol

But the probability of the node $\mathrm{K}$ will be small compared to the threshold value. So the routing protocol selects another path to broadcast the packets from the sender to receiver as shown in the above flow diagram.

In this algorithm, if the probability of a route is less than the threshold value (probability factor which we have assumed to be 0.8 that is $80 \%$ stronger route), then another route for forwarding the path is determined. The arrival rate of packet is calculated by finding the difference between current time and time when previous packet arrived. The service time is assumed to be a constant rate of $0.001 \mathrm{sec} /$ packets.

\section{ENHANCED PROBABILISTIC AD- HOC ON DEMAND ROUTING ALGORITHM}

Step1: Initialize network nodes Initialize the packet counter function Step2: Send Message to M Check Duplicate message M indicated Increase $\mathrm{c}$ value by 1

Step3: If(c <C) (i.e., C is the offset threshold) Set the forward probability to $\mathrm{P}$ 
Else

Step4: Message dropped on network (loss of packets)

Step5: Drop the message $\mathrm{m}$

Goto exit

Step6: Generate the random number as Rn more than the collection $[0,1]$

Step7: If $(\mathrm{Rn}<\mathrm{P})$

Message sending to broadcast

Broadcast the message $m$

Else

Step8: Drop the message $\mathrm{m}$

Exit

Step9: Every time update route information

\section{SIMULATION RESULT}

The simulation state is calculated particularly towards to evaluate the routine of probabilistic system direction-finding protocols. The network designed with 71 nodes more than a set four-sided Figure topology region of $1000 \times 1000 \mathrm{~m}$ by $5 \mathrm{~m} \mathrm{sec}^{-1}$ node velocity to 3 equal sources to destination relations. EPAODV have a quantity of simulate metrics used for the overall the proposed network direction-finding protocol.

\subsection{Comparison Result}

Table 3 shows the comparison results of PAODV with that of the EPAODV. The proposed approach shows a better result when compared with that of PAODV.

Table 2 shows the parameters are using in our work, we have take an different protocols,
Transmission range and Packet size based data transmission on the network.

\subsection{Throughput}

In the following Fig. 4 throughput performance of the network is explained.

\subsection{Packet Delivery Fraction}

The percentage of information received the target to generate by the sender node. It is planned as follows:

$$
\mathrm{PDF}=(\operatorname{Pr} / \mathrm{Ps}) \times 100
$$

where the Pr is total packet received and Ps is the whole packet sending on the process. The following Fig. 5 high light the performance of packet delivery ratio.

Table 2. Simulation process parameters

\begin{tabular}{ll}
\hline Parameters & Value \\
\hline Version & Ns-all in one 2.28 \\
Protocols & EPAODV \\
Area & $1000 \times 1000 \mathrm{~m}$ \\
Transmission range & $250 \mathrm{~m}$ \\
Traffic model & UDP, CBR \\
Packet size & 512 bytes \\
\hline
\end{tabular}

Table 3. Comparison results

\begin{tabular}{llllll}
\hline S. & $\begin{array}{l}\text { No of } \\
\text { nodes }\end{array}$ & Protocol & Throughput & $\begin{array}{l}\text { Average } \\
\text { delay }\end{array}$ & PDF \\
\hline 1. & 71 & PAODV & 3.84 & 22.90 & 94.76 \\
2. & 71 & Enhanced & 4.35 & 18.14 & 95.37 \\
& & PAODV & & & \\
\hline
\end{tabular}

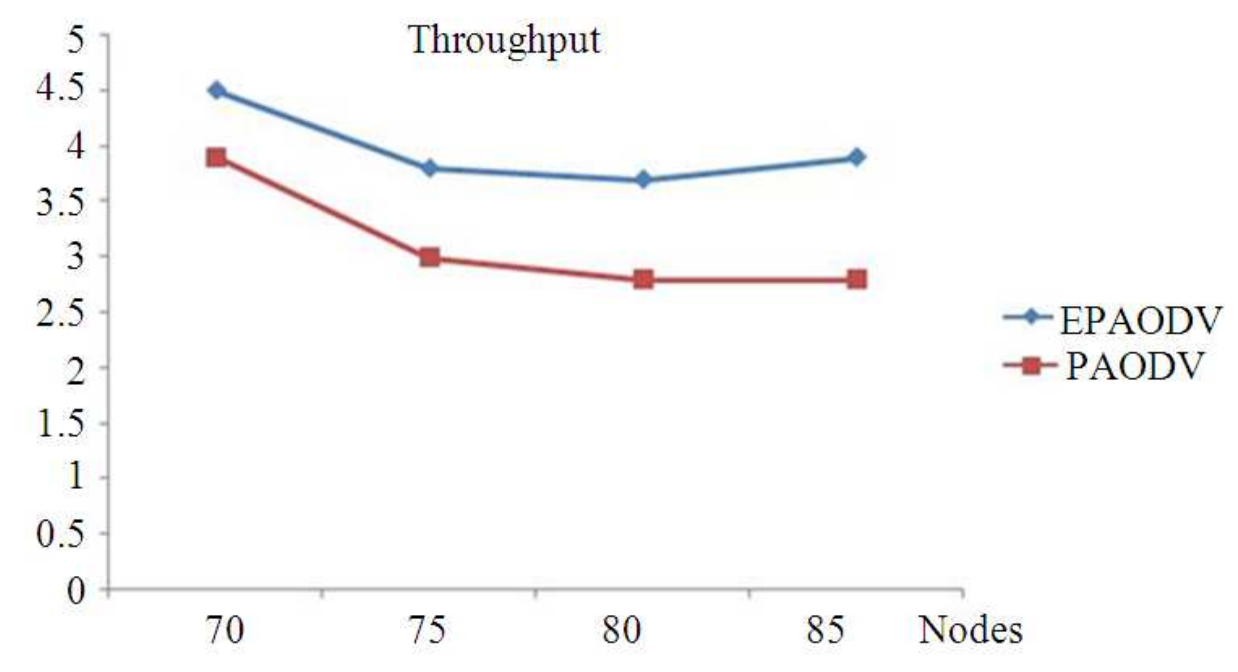

Fig. 4. Throughput ratio 


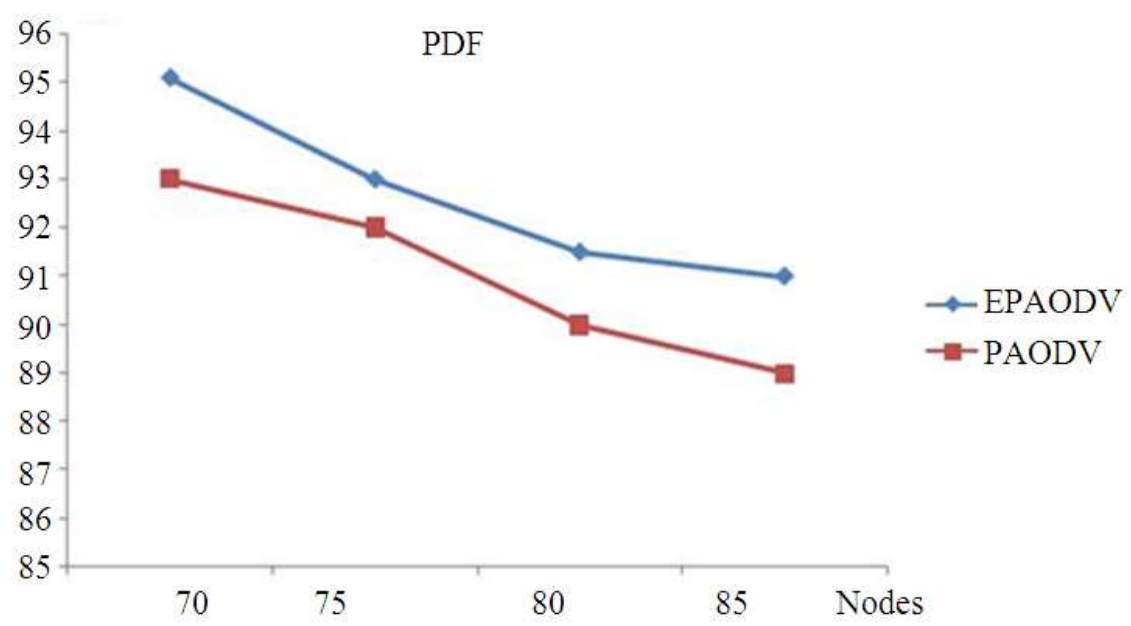

Fig. 5. Performance of packet delivery ratio

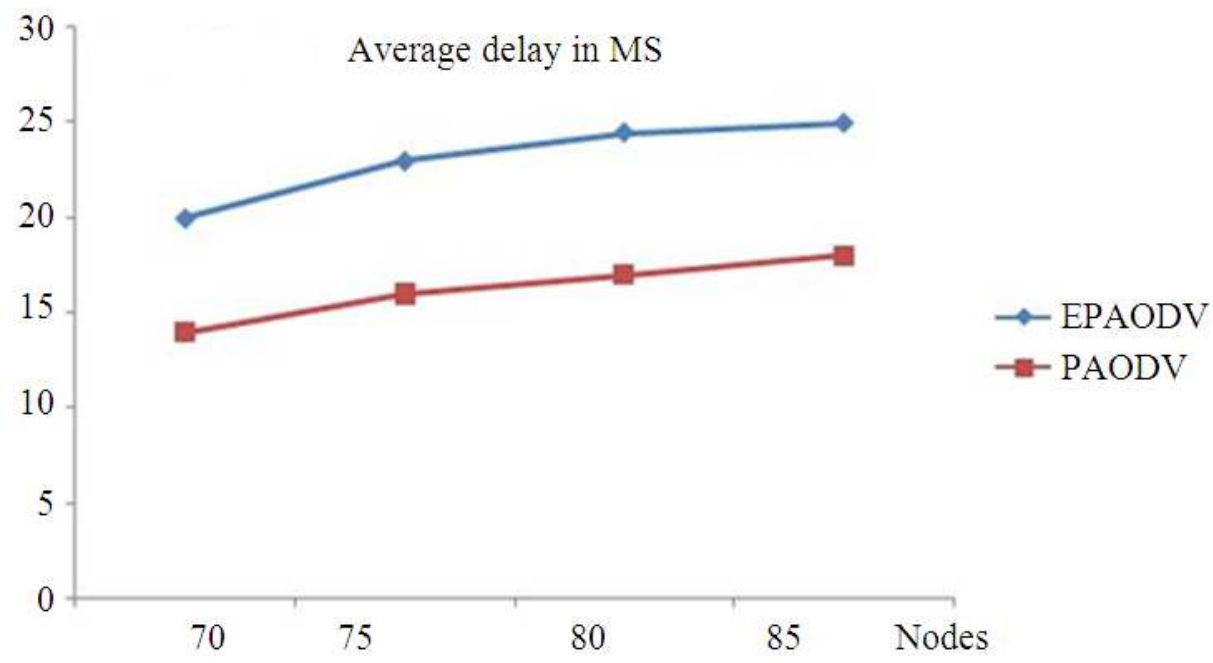

Fig. 6. Average end-to-end delay

\subsection{Average End to End Delay}

The end delay $\mathrm{D}$ is calculated jointly with all probable wait caused by storing capacity level through way finding performance, queuing at the boundary, another retransmit wait at Media Access Control (MAC), transmission level and then transmit's time. It is distinct as the occasion in use for a data to be sending across a MESH network in starting place to end:

$$
\mathrm{D}=(\mathrm{Tr}-\mathrm{Ts})
$$

where, $\mathrm{Tr}$ is receiver Time and $\mathrm{Ts}$ is sender Time.
Next to evaluate an end-to-end delay on sender node to receiver node of the position with existing and proposed routing protocol such as PAODV and EPAODV. The evaluation is explained through the following Fig. 6.

\section{CONCLUSION}

The present EPAODV protocol enhances the existing probability based distance vector directionfinding algorithm by changing queuing model which is used in the existing PAODV. By using the new EPAODV routing algorithm the performance of the 
networks has been determined. The results have been taken for throughput, data delivery ratio and then End delay. By comparing EPAODV with existing PAODV results show that our EPAODV protocol increases throughput, data delivery ratio and then decreases the end delay. Further we note if the network is high, the network performance like data delivery ratio is maximized and packet delay is minimized.

\section{REFERENCES}

Khandelwal, G., G. Prasanna and C. Hota, 2011. Probabilistic routing using queueing theory for MANAETs. Int. J. Wireless Mob. Netw., 3: 144-158.
Yassein, M.B. and M.B. Khalaf and A.Y. Al-Dubai, 2010. A performance comparison of smart probabilistic broadcasting of ad hoc distance vector. J. Supercomput., 53: 196-211.

Yassein, M.B. and M.O. Khaoua, 2007. Applications of probabilistic flooding in MANETs. Ubiquitous Comput. Commun. J. 